\title{
Evolución del concepto de desarrollo e implicaciones en el ámbito territorial: experiencia desde Cuba
}

\author{
Francisco ÁngEl BeCERRA LoIs \\ Jesús René Pino Alonso*
}

\begin{abstract}
This work examines some essential aspects about the evolution of the concept of development from the point of view of economic theory and its impact in regional economy. The emphasis is placed in the territorial inequalities and the job of the State to correct them, in particular within economies with the characteristics of Cuba, in which the role of the localities in their own integral development is crucial for the achievement of a more articulated and balanced territory. We offer some general guidelines to argue, scientifically and based on the current development theories, about the factors that underlie the dynamics of the socio-economical development in the scale of the territory-preferably in the locality sphereduring a reasonable time.
\end{abstract}

Keywords: development, socio-economical development, territory, local development, Cuba.

\section{Resumen}

Este trabajo examina aspectos esenciales sobre la evolución del concepto de desarrollo desde el punto de vista de la teoría económica y su reflejo en la economía regional. Se pone énfasis en las desigualdades territoriales y el papel del Estado para corregirlas, especialmente en una economía con las características del caso de Cuba, donde destacan la importancia que en los momentos actuales deben jugar las localidades en aras de lograr un territorio mejor articulado y equilibrado en su desarrollo integral. Se ofrecen pautas generales para argumentar con cientificidad y con base en las teorías del desarrollo cuáles son los componentes o factores que subyacen tras la dinámica de desarrollo socioeconómico a escala territorial-preferentemente en el ámbito de localidad-, durante un tiempo razonable.

Palabras clave: desarrollo, desarrollo socioeconómico, territorio, desarrollo local, Cuba.

* Facultad de Ciencias Económicas y Empresariales, Universidad de Cienfuegos "Carlos Rafael Rodríguez”. Correos-e: fbecerra@fcee.ucf.edu.cu y jrpino@fcee.ucf.edu.cu. 


\section{Introducción}

Las disparidades en el desarrollo generalmente son estudiadas desde un enfoque global, entre provincias o regiones, lo cual puede ocultar posibles desequilibrios en áreas de menor tamaño. Por tanto, es necesario analizar la evolución del nivel de desarrollo socioeconómico en la escala territorial, preferentemente con una desagregación menor, como es el caso de las localidades, para profundizar en el estudio de los problemas existentes en el interior de los territorios o provincias.

Para ello es muy importante conocer la evolución del concepto de desarrollo, desde su anterior consideración como sinónimo de crecimiento de la riqueza nacional, hasta su actual concepción como un fenómeno multidimensional y complejo, con un objetivo muy marcado en la búsqueda de la integralidad. Por consiguiente, para su medición y análisis es preciso incorporar a su estudio varias dimensiones.

En este trabajo pretende analizarse dicho concepto, su reflejo en la teoría económica y sus implicaciones para la economía regional. Se explicará la evolución doctrinal que ha experimentado la ciencia regional, con el énfasis en las desigualdades territoriales y en el papel del Estado, para finalmente analizar esos aspectos en el caso de Cuba y destacar la importancia de las localidades que integran cada territorio.

De modo que el objetivo general del artículo consiste en fundamentar teóricamente los aspectos esenciales que deben servir como pautas generales para argumentar con cientificidad y con base en las teorías del desarrollo cuáles son los componentes o factores que subyacen tras la dinámica de desarrollo socioeconómico territorial -provincia, región, municipio y preferentemente la localidad-durante un periodo razonable.

\section{Desarrollo}

Desarrollo es un término no sólo económico, aunque sí muy socorrido, traído y llevado en los últimos tiempos. Está presente, cada vez con más persistencia, en las reflexiones sobre economía. Empleado por académicos y políticos, por marxistas y neoliberales, se escucha lo mismo en un equipo de especialistas que lo estudia con la pretensión de agotarlo exhaustivamente, que en intercambios cotidianos entre conocidos. 
El concepto da título a actos prestigiosos: "Globalización y problemas del desarrollo"; a cursos especializados: "Maestría en desarrollo local"; a propuestas controvertidas: "Índice de desarrollo humano". En síntesis, parece ser un sustantivo muy versátil, cuyo empleo queda sometido a la intención de quien lo enarbola y a la interpretación del receptor del mensaje. Quizás esa riqueza de matices y la multiapropiación que se hace del término constituya el acicate para que no se detenga su estudio.

No pretendemos, por tanto, ni siquiera el intento de agotar el concepto. Sólo deseamos acotarlo en una dirección: ¿cómo hacer de ese concepto, que por veces se nos antoja 'etéreo', algo más concreto, más perceptible y, por qué no, más mesurable y visible?

En esto hay cuatro premisas importantes:

Premisa uno: Crecimiento no es igual a desarrollo; puede haber, excepcionalmente, crecimiento en ausencia de desarrollo; pero no puede haber desarrollo con ausencia de crecimiento. Más aún: la acumulación meramente cuantitativa de sucesivos crecimientos produce la transición cualitativa al desarrollo y lleva en sí a desarrollo.

Premisa dos: Desarrollo no es un término mesurable en términos absolutos (no hay 'desarrollo cero', no hay 'menos desarrollo'; su análisis estará siempre atrapado en dimensiones espacio-temporales que le otorgan relatividad a su expresión. Esto ocasiona que se exprese en término de niveles de desarrollo que encierran una relatividad, bien en el tiempo, bien en la dimensión geoespacial.

Premisa tres: Desarrollo es un fenómeno social e histórico, tanto porque su contenido es exclusivamente social, en tanto fenómeno, como porque por sus formas, expresiones y percepciones se manifiesta en una dimensión espacio-temporal determinada, que es reflejada por la conciencia social.

Premisa cuatro: Por tanto, desarrollo se refiere a niveles en el avance ascendente del individuo social, genéricamente considerado como ente humano, es decir, en sus relaciones sociales.

Estas cuatro premisas pueden parecernos muy abstractas, muy generales; pero sólo esa abstracción puede permitir al ascenso a lo concreto, vale decir, hacer más perceptible y mesurable el desarrollo.

El término desarrollo, como concepto, aparece por primera vez en un documento público en la primera Declaración InterAliada de 1941 y en la Carta del Atlántico del mismo año (Sunkel 
y Paz, 1986; Sunkel, 1996; Prats, 1999). Luego se reafirmó en la Conferencia de San Francisco en 1945 que dio origen a la Organización de Naciones Unidas (ONU).

En las ciencias económicas existen varias formulaciones que tratan de explicar este concepto, en dependencia del aspecto que cada teoría considera clave del desarrollo. ${ }^{1}$ Así, hay teorías basadas en las condiciones demográficas (Malthus y neomalthusianos); las condiciones geográficas y de dotación de recursos naturales (Landes, Sachs); la acumulación de fuerzas productivas (Harrod-Domar); la tecnología "exógena" (Ramsey, Solow); la tecnología "endógena” (Schumpeter, Romer, Lucas); las relaciones económicas internacionales (Gunder, Prebisch); las relaciones económicas internas: a) desigualdad (Easterly, Alesina), b) "dualidad estructural" (Lewis, Todaro), c) "demanda efectiva" (Rosenstein-Rodin, Hirschman), d) la superestructura jurídica y política (Coase, De Soto); la ideología y las tradiciones religiosas (Barro, Sala-i-Martin) (Romer, 1990; Huato, 2002).

Resulta conveniente realizar un comentario respecto a dichas teorías. Mientras las de Ramsey y Solow suponen una "economía competitiva", idealizada del mercado, las teorías de crecimiento endógeno requieren la participación del Estado. Debido a los beneficios externos que la innovación tecnológica difunde, los mercados subproducen investigación y desarrollo, por lo que se necesita que el Estado efectúe inversiones en estas áreas y en la acumulación del capital humano. Luego, en estas teorías se encuentra la génesis de las aportaciones de la economía regional en materia de desarrollo, pues "en bastantes aspectos, por no decir en la mayoría, la economía regional ha sido y es subsidiaria de las teorías económicas de carácter general" (Cuadrado, 1988: 70).

Hasta finales de los años sesenta del siglo xx, el concepto de desarrollo se confunde con los términos de 'crecimiento económico' y 'bienestar', medido en aquel entonces por el producto interno bruto (PIB), lo cual permitía clasificar a los países en más o menos desarrollados según los resultados de este indicador. Este enfoque, exclusivamente cuantitativo, es superado en los años setenta, cuando comienza a considerarse que para la medición del desarrollo deben existir condiciones necesarias que ga-

\footnotetext{
${ }^{1}$ Evidentemente no se explicará en detalle cada una de estas teorías, pero sí debe precisarse que de acuerdo con su concepción clave acerca del desarrollo, se han derivado importantes enfoques desde el punto de vista teórico y conceptual para la ciencia regional. Acerca de estas teorías, puede profundizarse en Robert et al. (1988).
} 
ranticen la realización del potencial humano (Seers,1970); y posteriormente se incorporó también la equidad, tratada como acceso a la ventaja (Colen, 1996). Surge en este periodo la idea del desarrollo como significado de un crecimiento estable del producto nacional bruto per cápita, pero no necesariamente todo crecimiento del producto nacional per cápita puede ser sinónimo de desarrollo. Sobre ello, Rodríguez (1983: 77) afirma críticamente: "Una economía puede crecer sin que avance hacia su real desarrollo. El desarrollo es una clase especial de crecimiento que asegura a un país crecer constantemente y a través de la autoimpulsión de su economía”.

Para lograr desarrollarse, los beneficios derivados del crecimiento deben distribuirse siguiendo patrones de equidad que eviten la marginalidad de grupos y capas de la población. Esto lleva implícita toda una concepción del desarrollo económico y social, lo cual ha sido explicado en Rodríguez y Carriazo (1987); Rodríguez (1983, 1990a y 1990b); Martínez (1991) y Zimbalist (1989).

La idea central remarca en la necesidad de establecer un vínculo orgánico entre los aspectos económicos y sociales del desarrollo, entendidos como una unidad integral con el objetivo de ayudar a los seres humanos haciéndolos más saludables, cultos, participativos y solidarios con los demás. ${ }^{2}$ En dicha concepción se aprecia una alta convergencia con el 'reemplazo' en la década de los noventa del concepto de desarrollo por el de desarrollo humano. Ello fue el resultado de los esfuerzos del Programa de Naciones Unidas para el Desarrollo (PNUD). Aquí, una visión del desarrollo enfocado a la producción material es sustituida por otra, centrada en las ampliaciones de las capacidades humanas. Junto a ello, surge una nueva forma de medición del desarrollo conocida como el Índice de Desarrollo Humano (IDH). Esta llamada dimensión humana del desarrollo se ha publicado en sucesivos informes del PNUD, el primero de los cuales se editó en 1990. Coincidentemente, en ese mismo año se publicó el Informe sobre el Desarrollo Mundial, dedicado a la pobreza, y Desafío para el Sur, de la Comisión Sur.

En las propias Naciones Unidas se ha propuesto la idea del desarrollo como un concepto formado por cinco elementos: ${ }^{3}$ la

\footnotetext{
${ }^{2}$ Se ratifica en la sinopsis elaborada por Osvaldo Martínez para la publicación de los resultados de la investigación CIEM-PNUD sobre Desarrollo Humano en Cuba (CIEMPNUD, 1997).

${ }^{3}$ Aparece en un Informe Oficial de la Secretaría General de las Naciones Unidas, firmado por el entonces secretario general Boutros Gali.
} 
economía como motor de crecimiento; la paz como fundamento del desarrollo; la justicia como pilar de la sociedad; el ambiente como una base para la sostenibilidad, y la democracia como base para una buena gobernabilidad. Contribuciones más recientes incorporan también algunos de estos elementos. Por ejemplo:

Economic development, as distinct from mere economic growth, must combine five elements: (1) self-sustaining growth; (2) structural change in patterns of production; (3) technological upgrading; (4) social, political and institutional modernization; and widespread improvement in the human condition (Adelman y Yeldan, 2000: 95).

\title{
Recientemente, en La Habana, el profesor Stiglitz (2002) ${ }^{4}$ re- afirmaba:
}

\begin{abstract}
Necesitamos tener objetivos claramente definidos. No se trata sencillamente de alcanzar un aumento en los ingresos, sino de crear un crecimiento económico equitativo, estable y democrático. Es importante que aumente el bienestar material, pero ello es sólo parte del objetivo. Necesitamos preocuparnos por la solidaridad social, la justicia, la calidad de vida y la educación [...] pues la educación no puede verse como un medio para aumentar los ingresos, sino también como un elemento que enriquece la calidad de vida.
\end{abstract}

Y, en su conferencia magistral, continuaba expresando más adelante:

La razón por la cual comencé mi exposición subrayando la necesidad de que establezcamos primero qué tipo de sociedad deseamos crear, y luego definamos cómo podemos utilizar la globalización en el logro de este tipo de sociedad, es porque de no hacerlo así perderemos la visión de lo que deseamos y como resultado confundiremos los medios con el fin.

El concepto de desarrollo pierde evidentemente su carácter estrictamente cuantitativo para transformarse en un concepto más cualitativo y, por consiguiente, más complejo, multidimensional e intangible. La integridad buscada aparece como una necesidad de hacer compatibles lo económico, lo social y lo ambiental, sin comprometer las posibilidades del desarrollo de las nuevas generaciones y de la vida futura del planeta. De esta manera, surgen los conceptos de 'desarrollo sostenible' y 'sostenido' que se cen-

${ }^{4}$ Premio Nobel de Economía por su trabajo compartido sobre análisis de los mercados con información asimétrica. Fue invitado al iv Encuentro de Globalización y Problemas del Desarrollo, celebrado en el Palacio de Convenciones de La Habana, en 2002. 
tran en la protección, conservación y uso racional de los recursos naturales (Nussbaum y Sen, 1996).

A continuación abordaremos dos cuestiones: las principales vertientes conceptuales del desarrollo en el espacio y la medición de las desigualdades en el territorio.

\subsection{El concepto de desarrollo en su dimensión espacial}

Apreciábamos anteriormente cómo el concepto de desarrollo se ha enriquecido con el tiempo; del mismo modo sucede al analizarlo en su dimensión espacial y surgen términos como desarrollo territorial, desarrollo regional, desarrollo local, desarrollo exógeno/endógeno, desarrollo descentralizado, cada uno de los cuales tiene su propia identidad.

El desarrollo territorial surgió como concepto asociado a la idea de territorio, entendido como superficie terrestre donde se asienta una población. Se vinculó, esencialmente, con escalas geográficas con diferentes cortes: país, región, provincia, comarca, municipio.

El término desarrollo regional es más completo, más abarcador. Ha sido definido como "un proceso localizado de cambio social sostenido que tiene como finalidad el progreso permanente de la región, de la comunidad regional como un todo y de cada individuo residente en ella" (Boisier, 1996: 33). Este desarrollo presupone además varios atributos simultáneos. ${ }^{5}$

En este concepto se distinguen tres dimensiones:

- Dimensión espacial: La consolidación del territorio como región.

- Dimensión social: La consolidación de la comunidad.

- Dimensión individual: Progreso de cada individuo como persona y como ente social.

\footnotetext{
${ }^{5}$ Primero, un creciente proceso de autonomía regional cada vez mayor para definir su propio estilo de desarrollo y para usar instrumentos de política congruentes con tal decisión. Segundo, una creciente capacidad regional para apropiarse de parte del excedente económico allí generado, a fin de reinvertirlo en la propia región y así diversificar su base económica y conferir sostenibilidad a largo plazo a su crecimiento. Tercero, un creciente movimiento de inclusión social, lo que implica, simultáneamente, una mejoría sistemática en la repartición del ingreso nacional entre las personas y una permanente posibilidad de participación de la población en las decisiones de competencia de la región; y, por último, una creciente autopercepción colectiva de 'pertenencia' regional; es decir, identificación de la población con la región.
} 
El desarrollo local ha recibido especial atención en los últimos años. Diversos autores de varios continentes como Vázquez (1997); Arocena (1997), Di Prieto (1999), Boisier (1996) y otros han propuesto sus propias definiciones, lo que hace difícil identificarse totalmente con alguna de ellas. Optamos por inferir algunos matices como son:

- Surge fundamentalmente en Europa como respuesta a las crisis macroeconómicas y se propaga como una experiencia de 'desarrollo desde abajo hacia arriba'.

- En América Latina surgen experiencias de base local.

- Constituyen aproximaciones teóricas conformadas a partir de la evidencia empírica.

- Es la expresión de una lógica de regulación horizontal.

- Constituye una posible respuesta a la crisis del modelo polarizado y a la reestructuración de la base económica: desagrarización, desindustrialización y terciarización.

- Se refiere a un espacio social enmarcado en una realidad territorial, en el cual las relaciones interpersonales, la cultura, las tradiciones y las costumbres juegan un papel importante.

- Emerge de la dialéctica global/local propia de la globalización.

- Potencia el desarrollo de los recursos humanos, económicos, físicos, naturales y socioculturales de la sociedad local con el fin de satisfacer a la sociedad humana y con ello aumentar la satisfacción de la sociedad local, basado esencialmente en la idea de la sostenibilidad.

- Presupone distintas dimensiones: económica, social, ecológica, institucional, cooperativa.

La respuesta local a los desafíos globales se instala mediante un conjunto de acciones de carácter muy diverso, que incluye los denominados hardware, software, orgware, ecoware y finware del desarrollo local (Vázquez, 1995).

El concepto de desarrollo endógeno resulta más controvertido y ha aparecido asociado al de crecimiento endógeno (Vázquez, 1997). ${ }^{6}$ Respecto a las definiciones de endógeno y exóge-

6 "La teoría del desarrollo endógeno se diferencia de la del crecimiento endógeno en que además del crecimiento de la producción, considerará las dimensiones sociales y medioambientales del desarrollo en que se adopta una visión territorial y no funcional de los procesos de crecimiento y cambio estructural, en que entienda que las formas de 
no, Boisier (1996) plantea que estarán muy vinculadas a la escala territorial. En el contexto de globalización y alta movilidad espacial del capital, el crecimiento territorial será más exógeno a medida que el recorte territorial sea más pequeño y los agentes que controlan los factores de crecimiento -acumulación de capital, de conocimiento, capital humano, política económica global, deuda externa- tienden a separarse de los agentes locales; los primeros agentes son quienes generalmente residen fuera del territorio en cuestión. Sin embargo, el mismo autor argumenta que el desarrollo debe considerarse como más endógeno debido a su asociación con la cultura local y sus valores.

El desarrollo endógeno podría entenderse como un proceso en el que se interceptan cuatro planos: el político, como creciente capacidad regional para tomar decisiones propias y definir un estilo de desarrollo propio; el plano de la endogeneidad económica, referido a la apropiación regional de parte del excedente económico para dotar de sostenibilidad el crecimiento y ampliar su base productiva; el plano científico y tecnológico, referido a la capacidad interna para realizar modificaciones cualitativas en el sistema, y finalmente la endogeneidad en el plano de la cultura como un factor de identidad socioterritorial (Boisier, 1996).

Finalmente, un concepto muy común ligado al desarrollo es también el de desarrollo descentralizado. Para autores como De Mattos (1990), Boisier (1990) y Solís (1999), implica en esencia aumentar el poder, la autonomía de decisión junto al control de los recursos, las responsabilidades y las competencias de las colectividades locales, todo ello en detrimento de los órganos del poder estatal central, lo cual constituye un enfoque eminentemente político y administrativo.

Ninguno de los conceptos hasta aquí analizados puede interpretarse de una manera aislada: hay una evidente intersección entre los conceptos de desarrollo territorial y desarrollo regional con los de desarrollo local, exógeno/endógeno y descentralizado. Todos deben conjugarse para propiciar opciones de desarrollo en diferentes dimensiones espaciales, con el objetivo de atenuar las desigualdades regionales, acerca de las cuales abordaremos algunos aspectos.

organización, los sistemas de relaciones y la dinámica de aprendizaje son los factores impulsores de la dinámica económica. Tiene, además, una visión más compleja de los mecanismos de acumulación del capital, lo que lleva a plantearse las políticas de desarrollo económico desde el territorio, de tal manera que la sociedad participe en la definición y el control de las acciones" (Vázquez, 1997: 126). 


\subsection{Principales vertientes conceptuales del desarrollo espacial. La medición de las desigualdades regionales}

Paralelo al surgimiento del concepto de desarrollo, emerge el de desigualdades regionales y, junto a él, los países y regiones comienzan a tomar mayor conciencia de las desigualdades espaciales existentes en sus territorios. Estas desigualdades, ya sean inter o intrarregionales, y los diferentes niveles de desarrollo son en el fondo la verdadera justificación para actuar en el territorio, y constituyen el aspecto definitorio de las políticas regionales, las cuales forman parte inseparable del tema de las desigualdades o desequilibrios regionales.

Es sabido que el crecimiento no aparece en todas partes a la vez, sino que se manifiesta en puntos o polos de crecimiento con intensidades variables (Perroux, 1955). El análisis de la dinámica territorial del desarrollo demuestra que no hay coincidencia de un proceso de desarrollo que se haya extendido simultáneamente sobre todo un territorio nacional, o un proceso de desarrollo que, surgido desde abajo, se haya diseminado instantánea y equilibradamente. Por eso estas desigualdades comienzan a ser corregidas mediante diferentes mecanismos, fundamentalmente mediante la intervención directa del Estado.

Desde su surgimiento, las políticas regionales -en sus vertientes de enfoque de redistribución o enfoque de compensaciónhan tenido como razón de ser la eliminación, disminución o reducción de los desequilibrios regionales y la necesidad de aplicar medidas favorables al desarrollo económico de las zonas más atrasadas. Se plantean dos problemas: el primero, asociado a la necesidad de desarrollar métodos de investigación que permitan cuantificar las desigualdades en cuanto a niveles de desarrollo o calidad de vida de los habitantes de una región y, el segundo, relacionado con el papel de los actores sociales involucrados en la implantación de las medidas correctoras que permitan atenuar dichas desigualdades (Cuadrado, 1988).

Respecto a la medición existe ya un consenso en la necesidad de considerar, además de las condiciones materiales, las inmateriales o intangibles para medir integralmente el desarrollo de los individuos sociales. Pero si es difícil medir aspectos cuantitativos, más difícil aún es medir los cualitativos. Debido a ello, los distintos enfoques sobre medición de los niveles de desarrollo utilizan indicadores objetivos, detrás de los cuales hay aproximaciones de medidas en el orden cualitativo. 
Autores como Pena (1977) y Zarzosa (1996) plantean tres enfoques para la medición del bienestar social: el contable, la función de utilidad y los indicadores sociales. Este último concibe el desarrollo como un enfoque multidimensional, a diferencia de los anteriores, en los que la variable ingreso es clave para la medición. En este sentido, son muy conocidos los trabajos de Kuznets (1955) sobre la hipótesis de la U invertida -la desigualdad primero crece, y luego decrece en la medida que se incrementa la renta per cápita- y los de Theil o Gini, que utilizan como medida de desigualdad índices basados en la renta per cápita (Todaro, 2000).

La concepción multidimensional se materializa a través de la inclusión de un conjunto de indicadores económicos y sociales en la medición del desarrollo. Ésta se inicia con la publicación del informe Definición internacional y medida de los niveles de vida del Consejo Económico y Social de las Naciones Unidas en 1960. Desde entonces ha predominado en organismos internacionales e instituciones públicas especializadas en el tema. El procedimiento se orienta a la determinación de componentes, factores o parcelas en las cuales puede dividirse el desarrollo y considerar indicadores de aspectos particulares sobre cada componente. La solidez en los fundamentos de estos componentes o factores permite cuantificar en forma razonable los resultados sobre los diferentes niveles de desarrollo y contribuye a explicar el origen de las desigualdades regionales.

En el siglo anterior se desarrollaron varias aportaciones teóricas en el campo de las teorías espaciales con una importante contribución a la explicación del tema de los desequilibrios interregionales. Sin pretender explicar detalladamente tales teorías, lo cual sería prácticamente inabarcable en este trabajo, sí es necesario expresar los grandes problemas que se ha planteado la ciencia regional, la cual se ha enriquecido de aportaciones procedentes de la economía del desarrollo, la economía del bienestar y del análisis económico general.

Convencionalmente, pudiera hablarse de dos planos o perspectivas de las teorías explicativas de la economía regional: la visión microeconómica, la cual aborda el problema de la localización y organización del espacio, y las teorías del crecimiento regional, que en esencia tratan de dar respuesta a las siguientes preguntas: ¿el espacio tiende a homogeneizarse o a diferenciarse?, ¿las diferencias interespaciales tienden a aumentar o a disminuir?, ¿por qué unos espacios crecen más que otros?, ¿̇uáles 
son los factores explicativos de las mayores o menores tasas de crecimiento de las diferentes regiones? (Mella, 1998). Alternativamente y de manera complementaria, en los últimos años ha adquirido gran fuerza el enfoque del desarrollo endógeno, que "concibe el espacio de manera activa, esto es, como territorio dotado de dinámica autónoma, agente de desarrollo económico y de transformación social (Friedmann, 1981; Furió, 1996)"7 (citado en Mella, 1998: 19).

El estudio del problema de la localización y organización del espacio es el más remoto en las doctrinas de la ciencia regional. Su incorporación a la teoría económica es fruto del esfuerzo de la escuela alemana de pensamiento, que se planteó cuatro modelos básicos: el modelo de Vön Thunen de 1826 o la teoría de la renta diferencial, que se proyecta del espacio rural al espacio urbano; el modelo de Weber de 1909, con una orientación hacia el transporte y que trata de explicar las localizaciones industriales; los modelos desarrollados por Hotelling en 1929 y Palander en 1936, sobre el duopolio espacial entre productores o las áreas de mercado, y finalmente los modelos de Christaller de 1933, con la teoría de los lugares centrales, y Lösh de 1940, con la región ideal. Posteriormente la escuela alemana fue relevada por diversas corrientes europeas y por una "poderosa escuela americana [liderada por Walter Isard]" (Mella, 1998: 14).

Los problemas espaciales no culminan con el análisis microeconómico de la localización. Como se explicó anteriormente, en las teorías del crecimiento regional se formulan otros problemas de gran importancia. En dependencia de las respuestas a las preguntas clave enunciadas con anterioridad, existen dos corrientes de pensamiento: las teorías de la convergencia regional y las teorías de la divergencia regional.

Las teorías de la convergencia regional plantean que las disparidades interregionales son transitorias, al final tenderán a desaparecer y se alcanzará un equilibrio estable más o menos prolongado, según el periodo de ajuste. Las teorías de la divergencia regional son contrarias: sostienen que las disparidades regionales no son transitorias, sino que forman parte de la propia naturaleza del crecimiento económico y mantienen el sistema en condiciones de desequilibrio. Postulan la organización desigual del espacio y suponen que es heterogéneo en la dota-

${ }^{7}$ Lo ratifica Mella (1998) en el capítulo primero de Economía y política regional en España ante la Europa del siglo XXI, titulado: "Evolución doctrinal de la ciencia regional: una síntesis". 
ción de recursos productivos y de factores económicos y extraeconómicos. En suma, plantean que las disparidades no sólo se reproducen con el tiempo, sino que tienden a agravarse.

Alternativamente a la llamada concepción funcional del espacio, abarcadora de las tesis de la convergencia y divergencia regional, se desarrolló en los últimos 20 años del siglo anterior, y continúa vigente, la perspectiva del desarrollo endógeno. Surgió como consecuencia de la crisis de la concepción funcional del espacio y su incapacidad para explicar las complejas estructuras productivas de los territorios, los nuevos comportamientos espaciales, la nueva noción del desarrollo y otros aspectos que adquirieron relevancia ante las grandes transformaciones que ocurrieron en el mundo con posteridad a la crisis de los años setenta. Esta renovación doctrinal está basada esencialmente en:

a) la nueva concepción del espacio (Vázquez, 1988); b) los modelos de desarrollo local (Vázquez, 1986, 1995, 1996; Albuquerque, 2001 y otros); c) el concepto de distrito industrial (Marshall, 1900; Becattini, 1992) y d) la tesis del 'entorno innovador' (Maillat, Quevit y Senn, 1993) (citado en Mella, 1998).

La nueva perspectiva, fundamentada en hipótesis menos abstractas y más realistas que las de los enfoques anteriores, ofrece pautas más razonables para la comprensión del desarrollo social, económico o socioeconómico, o más sencilla y precisamente: desarrollo, como avance ascendente de colectividades o comunidades humanas. Pero el propio concepto de colectividad, comunidad o grupo de seres humanos sigue siendo etéreo para el propósito.

Muy bien puede ilustrar el caso, también frecuentemente referido, del desarrollo de una nación. Varios indicadores suelen emplearse para evidenciarlo, argumentarlo o demostrarlo, según pretenda el expositor. Casi siempre aparecen cifras que hablan más de crecimiento que de desarrollo. Pero las medidas de una nación suelen ocultar grandes brechas entre sus extremos, que frecuentemente sobrepasan con creces las desviaciones estándares y llevan a cuestionar si es crecimiento o desarrollo.

Así resulta que la nación, como marco de referencia, deja muchas incógnitas y dudas para hablar más concretamente de desarrollo. Así también es válido para percibirlo y medirlo, por tanto, ha de serlo también para planearlo y conducirlo de modo concreto. 
Por otro lado, el desarrollo de una nación, comunidad o colectivo es alcanzable sólo a condición de que cada elemento integrante del conjunto, es decir cada individuo social, se desarrolle. El sistema social se desarrolla sólo si los individuos que lo componen logran ese ascenso sostenido de sus capacidades, calificación, interacción e integración.

Por lo tanto, el desarrollo no es etéreo, sino algo muy concreto: es el de cada individuo social particular, de las interacciones e interrelaciones con los demás semejantes, que lo hace conformar comunidades de distintas dimensiones, por cuyas interrelaciones logra avanzar, crecer, ascender, en fin, desarrollar al medio que lo rodea y que transforma, en cuyo proceso se autotransforma y autodesarrolla a través de su actividad vital distintiva: el trabajo.

La dimensión espacio-temporal en la que transcurre ese proceso es, a su vez, el espacio en el que existe; muta, transforma y ejecuta esta forma superior y más compleja del movimiento, es decir, la social. Ese espacio geofísico en que se camina a través del tiempo realizando la actividad vital de cada quien es el de la localidad en la que existimos, donde interactuamos realmente con los demás semejantes y el entorno.

Es en la localidad donde tienen su forma concreta los recursos, desde naturales hasta artificiales, pasando por los recursos humanos, las habilidades, destrezas y conocimiento, el aire y el paisaje, las infraestructuras productivas, de servicios, comunicación, etcétera, y las superestructuras de regulaciones, reglas, normas, leyes, organizaciones sociales, políticas, instituciones morales, profesionales, religiosas, los valores, costumbres, tradiciones... en fin: todos los componentes reales de la vida cotidiana y del desarrollo están presentes y se perciben en la localidad.

Si quiere hablarse de desarrollo en concreto, no en abstracto; si se quiere medir, percibir, plasmar, conducir, alcanzar, evidenciar, mejorar, disfrutar, no queda alternativa que la escala local, donde se hace visible, mesurable, planeable, concretable y dirigible.

La localidad, capaz de alcanzar integración de la cadena del valor, de mejorar los recursos propios, endógenos, para ese desarrollo, es la que interactúa con otras y con otros espacios, dentro o fuera de fronteras.

¿Quién produce?, ¿quién exporta?, ¿quién asegura calidad?, ¿quién alcanza competitividad?, ¿es el país, toda la nación o es la empresa? Está claro que en el fondo es la empresa, por mucho que se intermedie, regule o distancie de esos procesos. 
El país puede gozar condiciones que propicien o que obstruyan más o menos -según sea el sistema de gestión económica que se implante, sus regulaciones, leyes, formativas- la gestión eficiente de su tejido empresarial; pero son, en primera y última instancia, las empresas las que determinan la calidad, cantidad, actualidad tecnológica, capacidad de competencia, rentabilidad, oportunidad y demás rasgos que condicionan la posibilidad de ascender a niveles sucesivos, superiores y crecientes, de desarrollo.

Las condiciones inmediatas de desenvolvimiento, gestión, existencia, suministro de recursos -ante todo humanos, que son los decisivos- de las empresas están en la localidad a la que están articuladas de modo vital. De la localidad y su entorno no pueden prescindir.

En resumen: el desarrollo arranca, se concreta, se alcanza, se expresa, se percibe, se logra, tiene efectos y, en fin, existe como exponente del desenvolvimiento de la forma social del movimiento en la localidad. Su agregación e interacción con el desempeño de otras localidades -aledañas o distantes, nacionales o del resto del mundo- pueden dar criterios, juicios y magnitudes del desarrollo en escalas espaciales superiores del desarrollo y sus proporciones como son las de provincia, región, país o grupo de países. Sin esta premisa resultaría vana la pretensión de interactuar con un mundo global, ya que sólo resultaríamos, o bien 'llevados', o bien 'marginados' por ese mundo global.

\section{Desequilibrios interregionales y el papel del Estado}

Las relaciones entre las teorías esbozadas en este trabajo y las políticas regionales son evidentes. Por ejemplo, una política basada en la explotación intensiva de los recursos naturales se deduce de la teoría de la base de exportación; una política basada en la localización de grandes empresas industriales se desprende de la teoría de los polos de crecimiento, o una política favorecedora del entorno empresarial se deriva de los planteamientos del desarrollo endógeno. Por consiguiente, las políticas regionales serían voluntaristas si no tomasen en cuenta las teorías económicas regionales. En este imprescindible cordón umbilical entre economía y política regional sale a la luz con mucha fuerza el tema de los desequilibrios interregionales y el papel del Estado.

Respecto al tema de los desequilibrios interregionales, los economistas suelen situarse en uno u otro campo, de acuerdo con su afiliación a los planteamientos positivos o normativos. En 
el primer grupo, el 'espacio' no era considerado prácticamente, pues se planteaba que la desigualdad es temporal (Ohlin, 1933; Hagerstrand, 1967; Williamson, 1965, citado en Mella Márquez, 1998). ${ }^{8}$ Las desigualdades no serán un problema siempre y cuando permitan mejorar la situación de todos, incluidos los más pobres, por lo que se satisface el óptimo de Pareto. ${ }^{9}$ La política económica se concibe como un medio para asegurar el funcionamiento eficiente del mercado, y éste, per se, conducirá a un equilibrio del desarrollo regional. En el segundo grupo, se plantea como idea central la incapacidad del mercado para solucionar los desequilibrios, por lo que será necesaria una intervención. Destacados economistas como Myrdal (1959) sostienen que el mercado tenderá a favorecer a las regiones que alcanzan una primera ventaja -ventaja de llegar primero- por lo que el Estado deberá intervenir directamente con medidas correctoras.

Entre otros, avalan esta posición los aportes de Perroux (1955) y Boudeville (1968), quienes centraron sus estudios en la polarización de industrias o ciudades como polos de crecimiento; y en el modelo centro-periferia, derivado de las aplicaciones al análisis regional de los trabajos pioneros de Friedmann (1966). Ambas líneas de pensamiento coinciden en que es posible superar las desigualdades regionales: por la vía mercado o por la vía Estado.

Ha sido objeto de mucha discusión el papel que debe jugar el Estado en el desarrollo regional y en el impulso a la equidad interregional. El origen de esta literatura conocida como 'economía del sector público' o 'hacienda pública' se remonta a la teoría del equilibrio general de Kenneth Arrow y Gerald Debreu. Cada vez más se tiende a reconocer que el sector público, a través de sus políticas, debe guiar, corregir y complementar al mercado en algunos aspectos (Musgrave y Musgrave, 1992; Stiglitz, 1993). Los llamados "fallos del mercado"10 describen las diferentes circunstancias por las cuales la asignación alcanzada por el mercado no será eficiente en el sentido de Pareto. Entre ellos se encuentran: competencia imperfecta, monopolio natural, existencia de bie-

8 “[...] en las etapas iniciales el crecimiento es concentrado y en las de madurez desconcentrado, debido a la existencia de rendimientos decrecientes y que, en consecuencia, las disparidades regionales dan paso, con el tiempo, a un proceso de convergencia" (Williamson, 1965 en Mella, 1998: 21).

${ }^{9}$ Acerca de la eficiencia en el sentido de Pareto, puede consultarse en cualquiera de los textos clásicos de economía y microeconomía. Sugerimos Samuelson (1992), Pindyck y Rubineld (1998).

${ }^{10}$ No es objetivo aquí participar en la polémica sobre los "fallos del mercado", como por ejemplo lo hace Bowers (1990). 
nes públicos, exceso de efectos externos negativos o insuficiencia de efectos externos positivos, información asimétrica, ${ }^{11}$ problemas en el logro de determinados objetivos.

Luego, y según lo hasta aquí descrito, la intervención del Estado se justificará para mejorar la eficacia en el mercado y alcanzar asignaciones de recursos más deseables según criterios de equidad, y para estabilizar la economía. Cuadrado (1988) argumenta que los principios de compensación y redistribución de rentas entre las "regiones más ricas" y "regiones más pobres" han centrado la atención de las políticas regionales. Entonces, continúa vigente una vieja polémica teórica entre los que postulan que sin una intervención correctora por parte de las autoridades públicas no es posible reducir las disparidades entre las regiones (tesis de divergencia) y los que cuestionan la necesidad de la política regional apoyándose en los principios y derivaciones del modelo neoclásico para mantener posiciones favorables a la "convergencia económica de las regiones". La evidencia continúa indicando que, al no estar presentes criterios técnicos sólidos e integralmente fundamentados en las asignaciones de recursos, se debilita el impacto de las políticas regionales y aumentan las desigualdades interregionales.

Otro enfoque muy diferente se plantea cuando el mercado se subordina al Estado y por ende la única forma de corregir los desequilibrios territoriales es a través de cambios más profundos en el sistema de relaciones sociales.

\section{Desarrollo socioeconómico territorial en el contexto cubano}

Es necesario aclarar que las teorías enunciadas han surgido de la sistematización de una realidad, tanto institucional como socioeconómica, diferente de la construida en Cuba, y aun cuando pudiese resultar valioso inferir matices generales para explicar determinados comportamientos de un territorio cubano, es prácticamente imposible identificarse totalmente con alguna de ellas al intentar analizar la evolución del desarrollo socioeconómico en la escala territorial.

\footnotetext{
11 "Si bien los problemas inherentes a la imperfección de la información y la competencia, así como los asociados a la integridad del mercado, son importantes en los países desarrollados, estos problemas están más arraigados en los países subdesarrollados. Como resultado, las políticas aplicadas en demasiados casos no lograron generar crecimiento, ni pudieron reducir la pobreza, la desigualdad o la exclusión” (Stiglitz, 1993).
} 
Algunos aspectos esbozados anteriormente han merecido una atención especial en los proyectos socialistas y han sido objeto de análisis en el caso de Cuba, cuya economía está actualmente inmersa en una larga y difícil transición al socialismo partiendo de condiciones de subdesarrollo secular. Selectivamente, en determinados sectores del país se han formado y continuarán formándose relaciones económico-sociales con un carácter mixto y con la influencia decisiva de principios socialistas, lo que da pie a la formación de una economía mixta de transición al socialismo, que es sin duda el principal resultado de la actual reforma económica cubana y la base del nuevo modelo económico (Monreal y Rúa, 1994; Figueroa et al., 1995). En esta economía, el peso fundamental declarado es y seguirá siendo estatal, por lo que un gran reto para Cuba y el socialismo estará en redimensionar el sistema empresarial de modo que se garantice el éxito de la empresa estatal.

En este tipo de economía las disparidades regionales, muy sentidas desde abajo, sólo pueden ser medidas y manejadas conscientemente en los niveles superiores, desde donde es más procedente el establecimiento de políticas de corrección dirigidas a la equiparación, equidad y sostenibilidad de los territorios. Por tanto, las políticas regionales, cada vez más, deben poner énfasis en favorecer el desarrollo de las capacidades de crecimiento propias de cada región, más allá de la mera función compensatoria.

Desde el punto de vista macroeconómico, el proyecto socialista cubano se ha planteado dos objetivos fundamentales: alcanzar el desarrollo económico y construir una sociedad lo más equitativa posible. La evolución macroeconómica se ha caracterizado por distintos momentos económicos, los cuales han sido sistematizados por autores cubanos ${ }^{12}$ en varios periodos:

- Periodo 1959-1963: los grandes cambios.

- Periodo 1964-1967: reanimación económica.

- Periodo 1968-1970: zafra de los 10 millones.

- Periodo 1971-1975: crecimiento acelerado.

- Periodo 1976-1985: estabilidad macroeconómica.

- Periodo 1986-1989: desaceleración.

${ }^{12}$ Clasificación y sistematización realizada por Óscar U-Echevarría Vallejo, Alina Hernández Montero y Yenniel Mendoza Carbonell (U-Echevarría et al., 2002). 
- Periodo 1990-1993: crisis y asimilación del impacto externo.

- Periodo 1994 hasta la actualidad: adaptación, transformación y recuperación. ${ }^{13}$

En cada una de ellos se han mantenido inalterables los objetivos fundamentales planteados con anterioridad; sin embargo, debe destacarse que el impacto sobre el territorio no fue igual en cada momento y ha condicionado diferentes enfoques y etapas en la planificación territorial.

La necesidad de un modelo de desarrollo territorial surgió debido a las grandes desproporciones económicas que existían antes del triunfo de la Revolución de 1959. El municipio ocupó un lugar muy significativo en las nuevas concepciones que se plantearon. Hay que recordar que la tradición del municipio en Cuba se remonta a la época colonial y abarca todo el periodo republicano. Tanto en la Constitución de 1901 como en la de 1940, el municipio tuvo un lugar distintivo. La primera ley municipal se promulgó en 1902 y en la Constitución de 1940 se planteó la necesidad de revitalizar la actividad municipal.

Posterior a 1959, los primeros esfuerzos en la búsqueda de un mayor equilibrio espacial se orientaron hacia el ordenamiento de los territorios, con el objetivo de impulsar un conjunto de medidas derivadas del proceso revolucionario. La instauración en 1976 de los órganos locales del Poder Popular y de un subsistema municipal fue una acción importante en el proyecto de modernización del sistema político y estatal, que se denominó proceso de institucionalización. Su institución incrementó las facultades y atribuciones de las provincias y fortaleció la autoridad e importancia de los municipios, los cuales asumieron la administración de empresas y establecimientos que antes eran administrados por el poder central. De este modo se le facilitó el marco legal a los municipios para desarrollar con más flexibilidad la planificación y administración de actividades económicas y sociales.

La institucionalización incluyó varias acciones básicas, ${ }^{14}$ dos de las cuales fueron la implantación de una nueva División Polí-

13 "A partir de 1993 [...] comenzó un periodo recuperativo compuesto por dos etapas: 1994-1996 y 1997-1999" (Ferriol et al., 2003).

${ }^{14}$ Los autores recomiendan al lector el texto Participación popular y desarrollo de los municipios, donde se refiere a los antecedentes y características del diseño municipal cubano, con énfasis en las acciones básicas que supone la institucionalización (Dilla et al., 1993: 26). 
tica y Administrativa (DPA) y la implantación paulatina del Sistema de Dirección y Planificación de la Economía Nacional (SDPE).

La DPA tenía como objetivo dotar al país de una estructura regional más acorde con la evolución demográfica y con los planes de desarrollo. Para entonces existían en Cuba seis provincias, 58 regiones y 407 municipios. Con la nueva DPA se suprimió el eslabón regional, se redujo el número de municipios a 169 y se ampliaron a 14 el número de provincias. ${ }^{15}$ Por su parte, el sDPE fue concebido como un conjunto de normas, métodos y procedimientos a través de los cuales debían realizarse la organización, planificación, gestión y control del conjunto de la economía nacional.

Según el Informe Central del Primer Congreso del Partido Comunista de Cuba (PCC), el municipio adquiere nuevas atribuciones, con tendencia a una mayor descentralización, fortalecimiento de la autoridad e importancia económica.

\begin{abstract}
[A ellos] se encargará la administración de miles de unidades hasta ahora atendidas por los organismos centrales del Estado; la necesidad de dar a las nuevas instancias territoriales las características idóneas para facilitar la planificación y administración de las actividades económicas y sociales, de acuerdo con el Sistema de Dirección de la Economía, que demanda una adecuada relación entre centralización y descentralización de las decisiones, la participación de las masas en esta dirección y la más eficiente organización de la gestión económica (Informe Central, 1975: 161).
\end{abstract}

En el plan quinquenal de 1976-1980, se hizo especial hincapié en la necesidad de lograr una acertada distribución territorial de las fuerzas productivas entre las distintas zonas del país. La Junta Central de Planificación (Juceplan) consideró como una línea importante de su trabajo el perfeccionamiento de la planificación territorial, aspecto que se incluyó en el Anteproyecto de Indicaciones Metodológicas del Plan de la Economía Nacional.

En sentido general, durante el periodo transcurrido desde el año 1975 hasta finales de la década de los ochenta, se trabajó en el establecimiento de las bases organizativas y las condiciones necesarias para garantizar un desarrollo lo más equilibrado posi-

${ }^{15}$ Respecto a la DPA sus autores argumentan: "Ciertamente, la división políticoadministrativa puede considerarse un proyecto racional y realista que derivó en una consecuente política regional dirigida a reducir las disparidades de desarrollo, promover la integración e interdependencia regional y, al mismo tiempo, disminuir la brecha campo-ciudad de la cual resulta compromisoria. Sus diseñadores, sin embargo, no siempre pudieron evitar cierto disloque de las regiones socioculturales en función de una distribución demográfica y económica más equitativa y funcional a los planes de desarrollo" (Dilla et al., 1993: 31) 
ble de los territorios y la reducción de las disparidades intrarregionales. Se intentó que la planificación territorial pasara a una etapa superior a fin de garantizar la proporción debida en el desarrollo económico y social de los territorios, lo que permitiría una participación más activa de los órganos locales del poder popular y que los objetivos de desarrollo previstos en los planes se correspondieran con las decisiones que tomaran las provincias.

La estrategia general se orientó, entre otros aspectos, a considerar la integridad entre los aspectos económicos y sociales del desarrollo y a extender las políticas sociales a todo el territorio nacional, con énfasis en las provincias, en las ciudades intermedias o secundarias y en las zonas rurales, lo que favorecía la integración entre la ciudad y el campo.

Sin duda fue una etapa en la que se obtuvieron logros importantes, los cuales coexistieron con varias deficiencias que impidieron el éxito de las políticas regionales y retardaron el propio proceso de desarrollo y su evolución integral, al amenazar la obtención de los resultados esperados en determinados objetivos estratégicos. Entre las deficiencias podrían citarse: falta de recursos, escasa cooperación intraterritorial, falta de autonomía municipal, exceso de centralización económica y financiera, ausencia de una cultura de trabajo en la base para asumir la descentralización, limitaciones y errores del proceso inversionista; además, carencia de documentación teórica-metodológica, poca aplicación de técnicas de análisis regional, falta de preparación técnica y profesional de los técnicos en la base, limitaciones de la información estadística territorial, etcétera.

No obstante, el país logró una estabilidad en los principales indicadores globales de la economía. Tras 15 años de expansión en el crecimiento económico se presentó un periodo que tuvo dos momentos muy significativos: primero, la crisis económica de los años 1986-87, cuyos efectos recesivos se extendieron hasta 1989 , y el nacimiento de un proceso conocido como "rectificación de errores y tendencias negativas", y segundo, el derrumbe del sistema socialista europeo y particularmente del modelo eurosoviético en 1991, con terribles consecuencias para Cuba, que se agravaron de un modo especial en 1993, al punto que quizá muy pocas economías contemporáneas hayan experimentado tan brusca afectación en sus principales agregados macroeconómicos y el inicio de una significativa etapa caracterizada por medidas de ajuste y reforma. 
[La crisis de los años noventa] no sólo dejaba al descubierto fenómenos que demandaban una atención prioritaria y ponían en tela de juicio la concepción del modelo de desarrollo aplicado hasta ese momento, sino que amenazaba la conservación del proyecto revolucionario mismo (CIEM-PNUD, 1997: cap. II, "La estrategia de Cuba para el desarrollo humano").

$\mathrm{Al}$ periodo caracterizado por medidas de ajuste y reforma se le conoce en el país como "periodo especial en tiempo de paz" (Figueroa et al., 1995). Se trató entonces de preservar la concepción de desarrollo referida en la investigación, en un contexto de extremas tensiones económicas y en medio de un escenario internacional caracterizado por un creciente proceso de globalización y apertura externa.

Durante estos años se desarrollaron varias medidas de ajuste funcional como respuesta a la crisis externa (U-Echeverría et al., 2002), las cuales condicionaron importantes transformaciones económicas y sociales como: despenalización de la tenencia de divisas, apertura a la inversión extranjera, reorientación geográfica y descentralización del comercio exterior, políticas de ampliación del empleo por cuenta propia, cooperativización de la actividad agropecuaria con la instauración de las UBPC, proceso de redimensionamiento empresarial, saneamiento económico y financiero, apertura de los mercados agropecuarios, de bienes industriales y artesanales, puesta en marcha de nuevos programas sociales y una gradual descentralización en la toma de decisiones y en la autonomía territorial para la búsqueda de soluciones, entre otras. En sentido general, la adaptación de la estrategia de desarrollo económico y social se orientó a la concentración y distribución de los escasos recursos en función de una política social conducida por el Estado y con una amplia prioridad a los servicios sociales básicos, a pesar de la gran contracción económica. ${ }^{16}$

Las transformaciones, no finalizadas aún, han hecho más complejo el objeto de planificación, pero al mismo tiempo han favorecido a los territorios con una gradual descentralización y mayor exigencia para insertarse en la elaboración de estrategias de desarrollo. Condicionaron, además, el surgimiento de nuevos actores, la disminución de la estructura de la administración pública, la fundación de nuevas entidades de servicios, el aumento del mercado interno y su segmentación y la descentralización de

${ }^{16}$ Acerca de los principios fundamentales de la política cubana en la esfera social puede consultarse el Informe Nacional de la Cumbre Mundial sobre Desarrollo Social de Copenhague. 
la gestión empresarial, entre otros aspectos. Por consiguiente, se hizo necesario asumir "[...] un papel cada vez más activo en la búsqueda e implementación de soluciones relacionadas con el desarrollo local”, según afirma la Resolución Económica del v Congreso del PCC. El territorio adquiere un papel muy relevante respecto a la vigencia de la planificación, la necesidad de adoptar un enfoque prospectivo en la planificación territorial y la formulación de estrategias emanadas del potencial endógeno subyacente en las localidades (García Peyán, 1997; González Gutiérrez, 2001).

Debe destacarse que existen diferencias notables entre las estrategias de desarrollo local que se han puesto en marcha internacionalmente y las experiencias que se han seguido en Cuba, tanto desde el punto de vista de la forma de movilización y combinación de los recursos de acuerdo con los objetivos del desarrollo local, como en la propia articulación de estrategias, la forma de gestionar el desarrollo y el proceso de planificación, entre otros. No debe descuidarse que cuando se trata de utilizar la capacidad endógena de los territorios, es muy importante considerar el punto de partida de cada cual.

Las nuevas condiciones exigen una combinación entre las potencialidades del territorio, la competitividad regional, los mecanismos de regulación estatal y de redistribución de la riqueza para mantener el propósito de avanzar en la reducción de las disparidades territoriales (González Fontes et al., 2002) como alternativa viable para disminuir la brecha en términos de desarrollo entre los territorios y en su interior, pues a nuestro juicio este es uno de los principales problemas que deberá continuar enfrentando la economía cubana en perspectiva.

La crisis de los noventa no permitió continuar al ritmo de años anteriores la política de igualación entre los diferentes territorios del país, pues el impacto de la globalización y la reinserción en los mercados mundiales contribuyó a reforzar las tendencias de selectividad y diferenciación espacial y entrañó grados superiores en la descentralización empresarial y territorial, a lo que habría que añadir la ausencia de una concepción acabada y sistematizada del desarrollo regional centrada en las potencialidades reales del territorio.

Las transformaciones han ido cambiando paulatinamente el diseño original de los gobiernos municipales: de una propuesta inicial centralizadora a un nuevo escenario territorial, donde los municipios tienen nuevas funciones económicas y requieren re- 
formular la concepción, organización y funcionamiento de la administración pública. La reformulación impone la necesidad de reconocer la municipalidad como una de las células básicas de la ciudadanía, y el gobierno municipal como la institución gestora del desarrollo local con mayores prerrogativas para satisfacer necesidades y deseos de la población, sin descuidar la articulación coherente con el modelo de desarrollo socioeconómico adoptado por el país.

La diferenciación espacial también se ha agudizado dentro de las provincias y entre zonas geoeconómicas, municipios, asentamientos poblacionales y otras escalas territoriales de menor dimensión.

\section{Medición y análisis del desarrollo socioeconómico en la escala territorial}

En este contexto cobra especial importancia caracterizar los componentes o factores que subyacen en la dinámica del desarrollo socioeconómico, desde la óptica de un territorio y sus unidades menores, pues el territorio, cada vez más, demuestra que es una unidad fundamental para medir, analizar y evaluar el proceso de desarrollo económico y social y validar con cientificidad su evolución en un periodo razonable.

Para estudiar el desarrollo regional en su totalidad y extraer conclusiones válidas, deben analizarse por separado los elementos que lo conforman, esto es, dividir el todo en partes y posteriormente sintetizar los aspectos más relevantes. Por eso a partir de la experiencia empírica obtenida en investigaciones realizadas por los autores, sugerimos definir al menos tres dimensiones, conscientes de la estrecha interrelación entre ellas y de las limitaciones respecto a su propio alcance.

Las dimensiones ${ }^{17}$ mínimas propuestas son:

- Dimensión económica y espacial (DEE).

- Dimensión demográfica y laboral (DDL).

- Dimensión social (DS).

A partir de ellas es posible centrar la atención en determinados componentes o parcelas con cierta homogeneidad, al tiempo que

\footnotetext{
${ }^{17}$ En varios estudios e investigaciones consultadas se presenta el estudio del desarrollo como un proceso que requiere un conjunto de dimensiones que interactúan entre sí (CIEM-PNUD, 1997; Segnestam, 2002; Herrero y Figueroa, 2001).
} 
pueden definirse áreas temáticas e indicadores, los cuales permiten acotar el objeto investigado con mayor precisión. Este aspecto merece un cuidadoso estudio y debe ser sometido al criterio de expertos, pues es uno de los más complejos por varias razones, una de ellas es la disponibilidad de la información deseada por los investigadores y su correspondiente homogeneidad.

La dimensión económica y espacial es fundamental, pues determina directamente el nivel de vida de la población al contemplar aspectos como el potencial de recursos naturales, su utilización, el capital disponible, el propio empleo, la infraestructura, el desarrollo tecnológico, las posibilidades de producciones propias, el espacio y el medio como factores esenciales para la economía de una región. La inclusión del espacio como un elemento clave en la economía es una de las razones esenciales que justifican el auge de la economía regional.

La dimensión demográfica y laboral también es básica debido a que considera aspectos relacionados directamente con la persona como son: tamaño de la población, concentración, evolución, comportamiento, ocupación, empleo, etc.; todos ellos vitales para comprender cuestiones sustanciales del desarrollo y el bienestar social en el plano territorial.

La dimensión social es muy amplia, incluye aspectos básicos y complementarios, entre los que pueden relacionarse: la salud, la educación, la vivienda, la justicia, la participación, la recreación, la libertad y la seguridad, entre otros.

En diversos trabajos consultados, que han sido dirigidos al estudio de la medición del desarrollo regional y las desigualdades territoriales, suele escogerse o sugerirse un periodo de estudio extenso (Estivill y Batista, 1985; Baró et al., 1988; Clavero et al., 1988; Rodríguez, 1988; Gordo, 2003; Herrero y Figueroa, 2001; Perón et al., 2001; Méndez, 2001; Colarte y Becerra, 2003). Sin duda, la extensión del periodo de estudio potencia la credibilidad de los resultados -especialmente si son utilizados en técnicas estadísticas univariables y multivariables- a riesgo de enfrentar la naturaleza dinámica y cambiante de las políticas socioeconómicas territoriales que, a menudo, distorsionan los datos y complican las comparaciones necesarias.

Por consiguiente, la medición y análisis del desarrollo socioeconómico regional, desde el punto de vista de la investigación empírica, es un clásico ejemplo de fenómeno multivariable, debido a que es un reflejo de varias características. La tendencia de los expertos es considerar el mayor número posible de indicadores 
interrelacionados para garantizar que no haya pérdida de información relevante. No obstante, es conocido que el manejo excesivo de indicadores puede acumular información redundante y dificultar el proceso de análisis. Evidentemente no pretendemos exponer aquí experiencias prácticas y muy valiosas en tal dirección, pero sí resulta conveniente plantearlo como experiencia metodológica debido a que constituye una forma de aproximarnos cuantitativamente a los complejos aspectos que hemos abordado desde un enfoque eminentemente cualitativo.

En el orden práctico, el diseño de la investigación responde a un análisis exploratorio. Es decir, se persigue descubrir las relaciones socioeconómicas existentes en la matriz de información espacial, que permiten revelar o aproximarnos a las tendencias del desarrollo socioeconómico territorial que mejor describa o se ajuste a la historia de la unidad objeto de estudio. En un segundo momento, y teniendo en cuenta el resultado anterior, se construyen índices de desarrollo de corte intermunicipal, que permiten, a partir de la observación de su evolución, clasificar en rangos el desarrollo socioeconómico relativo municipal y ordenar los territorios en el periodo seleccionado con base en la clasificación construida. Esta idea se expone en el cuadro 1, que permite apreciar la secuencia lógica del procedimiento metodológico propuesto según los siguientes resultados esperados:

\section{- Análisis por dimensiones, áreas temáticas y global}

Mediante el método de análisis y síntesis se definen las dimensiones, a cada una de las cuales se le aplica el método de componentes principales para determinar los factores subyacentes y explicativos del desarrollo socioeconómico. El análisis global constituye una síntesis de los resultados obtenidos en cada dimensión.

- Ordenamiento del territorio mediante la utilización de índices

Se construyen índices: parciales por cada dimensión; combinados, interrelacionándolos, y uno global -IG o IDM- integrándolas, lo que permite establecer una jerarquía en cada uno de los casos. Su interpretación ofrece una primera aproximación de los desequilibrios intermunicipales y locales.

\section{- Formación de grupos asociados al ordenamiento}

Mediante la utilización del análisis cluster se forman grupos, que además contribuyen a confirmar los resultados obtenidos en la jerarquía. 


\section{Cuadro 1 \\ Procedimiento metodológico propuesto}

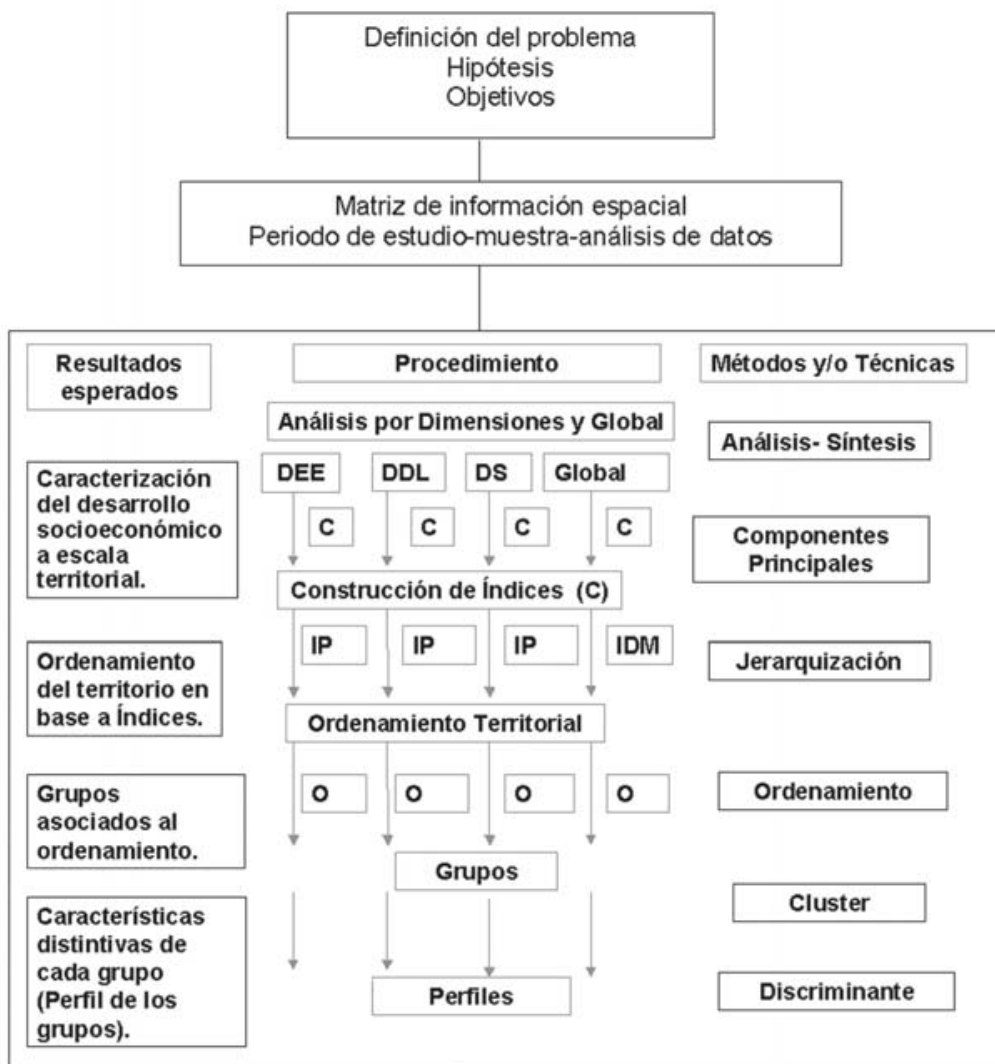

Evolución histórica del desarrollo socioeconómico en escala territorial Análisis comparativo global y validación de resultados

C: Componentes principales o factores.

O: Ordenamiento territorial.

IP: Índices parciales.

IDM: Índice global.

DEE: Dimensión económica y espacial.

DDI: Dimensión demográfica y laboral.

DS: Dimensión social.

Fuente: Elaboración propia. 
- Características distintivas de cada uno de los grupos

A través de la técnica de análisis discriminante se identifican cuáles son los componentes más distintivos que caracterizan a cada uno de los grupos.

- Evolución histórica del desarrollo socioeconómico a escala territorial

Finalmente, integrando los aspectos anteriores, es posible establecer una sistematización de la evolución del desarrollo socioeconómico en la escala territorial en el periodo de estudio para las unidades menores estudiadas.

\section{Conclusiones}

- El concepto de desarrollo aplicado a la economía regional es una derivación de las teorías económicas de carácter general. En el caso de Cuba, se plantea la necesidad de lograr un desarrollo lo más equilibrado posible entre los territorios y la reducción de las disparidades intrarregionales, con énfasis en la idea central de establecer un vínculo orgánico entre los aspectos económicos y sociales del desarrollo, y colocar en el centro de atención a los seres humanos.

- La evolución doctrinal de la ciencia regional demuestra que uno de los objetivos básicos de las políticas regionales continúa siendo la reducción de las disparidades interregionales y el impulso al desarrollo de las áreas atrasadas. Para ello el papel del Estado es importante en cualquier tipo de economía, especialmente en una economía mixta de transición al socialismo.

- Unidades importantes para la medición de los desequilibrios intrarregionales son los municipios y las localidades, y en el caso cubano constituyen las unidades menores de tipo regional sobre las cuales es posible recopilar un volumen de información útil para medir, analizar y validar la evolución del desarrollo en un tiempo razonable.

- La medición y análisis del desarrollo socioeconómico regional es un ejemplo clásico de fenómeno multivariable, debido a que es un reflejo de varias características, las cuales pueden ser sintetizadas en componentes o parcelas, que a su vez sean capaces de mostrar la dinámica de una batería de indicadores. Por eso proponemos para desarrollar investigaciones empíricas sobre estos aspectos la utilización de una estrategia metodológica basada en el empleo de técnicas estadísticas multivariables y validar los resultados finales de acuerdo con 
los componentes o factores identificados como explicativos de la evolución del desarrollo socioeconómico del municipio o localidad durante el periodo de estudio.

\section{Bibliografía}

Adelman, I. y E. Yeldan (2000), "Is This the End of Economic Development?", Structural Change and Economic Dynamics, núm. 11, Estados Unidos.

Arocena, J. (1997), "Lo global y lo local en la transición contemporánea”, Cuaderno del CLAEH, núms. 78-79, España.

Baró Llinás, J. et al. (1988), "Estratificación económica de Baleares. Un enfoque parcial”, Revista de Estudios Regionales, núm. 21, España.

Boisier Etcheveny, S. (1990), La descentralización. Un tema difuso y confuso, Instituto Latinoamericano de Planificación Económica y Social (ILPES), Documento 90/05, Santiago de Chile.

(1996), "Modernidad y territorio", Cuadernos del ILPES, publicación de Naciones Unidas, Santiago de Chile.

Boudeville, J. (1968), L'espace et les poles de croissance, Presses Universitaires de France, París.

Bowers, J. (1990), "The Environmental Crisis and the Limits of the Market", Discussion Paper 90/1, School of Business and Economic Studies, Universidad de Leeds.

CIEM-PNUD (1997), Investigación sobre el desarrollo bumano en Cuba, 1996, Caguayo, La Habana.

Clavero, A. et al. (1988), "Aproximación a la renta familiar disponible en las comarcas andaluzas", Revista de Estudios Regionales, núm. 21, España.

Colarte Morando, T. y F. Becerra Lois (2003), "Características subyacentes en el desarrollo socio-económico de la pro- 
vincia de Cienfuegos durante el periodo 1987-2000”, Economía y Desarrollo, núm. 2, La Habana.

Colen, I. (1996), "Igualdad de qué”, en M. C. Nussbaum y A. Sen (comps.), La calidad de vida, Fondo de Cultura Económica, México.

Cuadrado Roura, J. R. (1988), "Políticas regionales: hacia un nuevo enfoque”, Papeles de Economía Española, núm. 35, FFIES, Madrid.

De Mattos, C. (1990), "La descentralización, ¿̇una nueva panacea para impulsar el desarrollo local?", Revista de Estudios Regionales, núm. 26, España.

Di Prieto, L. (1999), El desarrollo local. Estado de la cuestión, Flacso, Buenos Aires.

Dilla, H. et al. (1993), Participación popular y desarrollo de los municipios, Centro de Estudios sobre América, La Habana.

Estivill, X. y J. M. Batista (1985), “Delimitación de regiones homogéneas para la elaboración del plan territorial de Cataluña mediante técnicas de análisis multivariante”, Ix Reunión de Estudios Regionales. Crisis, Autonomía y Desarrollo Regional, tomo III, Asociación Galega de Ciencia Rexional, Universidad de Santiago de Compostela, España.

Ferriol, A. et al. (2003), "Promoción de exportaciones, pobreza, desigualdad y crecimiento. El caso de Cuba en los noventa”, publicación electrónica El INIE en el 2003, La Habana.

Figueroa Albelo, V. et al. (1995), "Reforma económica en Cuba y sus direcciones principales", Contrapunto, La Habana.

Friedmann, J. (1966), Regional Development Policy: A Case Study of Venezuela, MIT Press, Cambridge, Massachusetts.

García Peyán, C. (1997), "Estrategia y territorio. Reflexiones sobre algunos temas clave en la planificación territorial”, Cuba: Investigaciones Económicas, núm. 1, Instituto Na- 
cional de Investigaciones Económicas de Cuba (INIE), La Habana.

González Fontes, R. et al. (2002), "La gestión del desarrollo regional en Cuba. Un enfoque desde la endogeneidad", Economía, Sociedad y Territorio, vol. III, núm. 12, julio-diciembre, El Colegio Mexiquense, A.C.

González Gutiérrez, A. (2001), "Vigencia de la planificación”, Cuba: Investigaciones Económicas, núm. 4, INIE, La Habana.

Gordo Gómez, P. (2003), "Balance de la ejecución de la iniciativa comunitaria Leader II en Castilla y León (1995-1999)", en O. Ogando Canaval y B. Miranda Escobar (coords.), Evaluación de programas e iniciativas comunitarias: Experiencias, nuevas orientaciones y buenas prácticas, Instituto de Estudios Europeos, Universidad de Valladolid.

Herrero Prieto, L. C. y V. F. Figueroa Arcila (2001), "Metodología para evaluar el desarrollo económico en unidades territoriales menores: una aplicación comparada a los casos de Castilla y León (España) y la Región de los Grandes Lagos (Chile)", investigación financiada por la Consejería de Economía y Hacienda de la Junta de Castilla y León (Orden del 15 de diciembre de 1989), Valladolid, España, Valdivia, Chile.

Huato, J. (2002), Curso de desarrollo económico on-line, State University of New York-Purchase College, http://www. geocities.com/juliohuato/sunny-p/6.htm.

Informe Central al I Congreso del Partido Comunista de Cuba (1975), Departamento de Orientación Revolucionaria del Comité Central, La Habana.

Kuznetz, S. (1955), "Economic Growth and Income Inequality", The American Economic Review, vol. XLv, núm. 1, Estados Unidos.

Martínez, O. (1991), "Desarrollo humano. La experiencia cubana”, Cuba Económica, La Habana. 
Mella Márquez (1998), "Evolución doctrinal de la ciencia regional: una síntesis", Economía y Política Regional en España ante la Europa del siglo XXI, Akal Textos, Ediciones AKAL, Madrid.

Méndez Delgado, E. (2001), "Planificación del desarrollo territorial en Cuba. Aplicación de técnicas de análisis regional para el diagnóstico en Villa Clara”, tesis doctoral, Santa Clara, Cuba.

Monreal, P. y M. Rúa del Llano (1994), "Apertura y reforma de la economía cubana: Las transformaciones institucionales”, Cuadernos de Nuestra América, La Habana.

Musgrave, R. A. y P. B. Musgrave (1992), Hacienda pública: teórica y aplicada, Mc Graw Hill, España.

Myrdal, G. (1959), Teoría económica y regiones subdesarrolladas (versión original en inglés de 1957), Fondo de Cultura Económica, México.

Nussbaum, M. C. y A. Sen (1996), La calidad de vida, Fondo de Cultura Económica, México.

Pena Trapero, J. B. (1977), Problemas de la medición del bienestar y conceptos afines: Una aplicación al caso español, Instituto Nacional de Estadísticas, España.

Perón Delgado, E. et al. (2001), "Un modelo social-territorial para los municipios de la provincia Camagüey (Cuba)", Economía y Desarrollo, núm. I. vol. 128, enero-junio, La Habana.

Perroux, F. (1955), "Note sur la notion de pôle de croissance", Économie Appliquée, núm. 7.

Pindyck, R. y D. Rubineld (1998), Microeconomía, 4ª ed., Prentice Hall, Iberia, Madrid.

Prats, J. (1999), La construcción histórica de la idea de desarrollo, http: ||wwwliigov.org \pnud \bibliote\7.htm. 
Robert, L. et al. (1988) "On the Mechanics of Economic Development”, Journal of Monetary Economics, núm. 22, pp. 3-42.

Rodríguez, C. R. (1983), Letra con filo, tomo II, Ciencias Sociales, La Habana.

Rodríguez, J. L. (1990a), Estrategia de desarrollo socio-económico, Ciencias Sociales, La Habana.

(1990b), "Cambio en la política económica y resultados de la economía (1986-1989)", Cuadernos de Nuestra América, julio-diciembre, La Habana.

y G. Carriazo (1987), Erradicación de la pobreza en Cuba, Ciencias Sociales, La Habana.

Rodríguez Rodríguez, V. (1988), "La medición de los desequilibrios territoriales en España”, Revista de Estudios Regionales, núm. 21, España.

Romer, P. (1990), “Endogenous Technological Change”, JPE, octubre, parte 2, S71-S102, Estados Unidos.

Samuelson, P. A. (1992), Economía, s. n., La Habana.

Seers, D. (1970), “The Meaning of Development”, Revista Brasileira de Economía, vol. 24, núm. 3, Brasil.

Segnestam, L. (2002), Desarrollo de indicadores. Lecciones aprendidas de América Central, http://www.ciat.egiar.org/indicadores/index.htm.

Solís, O. (1999), Metamorfosis del Estado y la política: del poder central al poder local, http: //www//iigov,org/pnud/bibliote/7.htm.

Stiglitz, J. E. (1993), El papel económico del Estado, Ministerio de Economía y Hacienda, Instituto de Estudios Fiscales, Madrid.

(2002), conferencia magistral: Asimetrias e hipocresía, La Habana, http:www//eleconomista/cubaweb.cu. 
Sunkel, O. (1996), El concepto de desarrollo, ILPES, Santiago de Chile.

y P. Paz (1986), El subdesarrollo latinoamericano y la teoría del desarrollo, 20ª ed., Siglo xxi Editores, México.

Todaro, M. (ed.) (2000), Economic Development, $7^{\mathrm{a}}$ ed., Addison-Wesley, Nueva York.

U-Echevarría Vallejo, O., A. Hernández Montero e Y. Mendoza Carbonell (2002), Antecedentes macroeconómicos, capítulo 3: "Aspectos globales. Estructura económica de Cuba”, tomo 1, editorial Félix Varela, La Habana.

Vázquez Barquero, A. (1995), "Desarrollos recientes de la política regional. La experiencia europea”, Desarrollo Económico Local, núm. 17, Universidad Autónoma de Madrid-Universidad de Vigo, España.

(1997), “¿Crecimiento endógeno o desarrollo endógeno?”, Cuadernos del CLAEH, núm. 78-79, España.

Zarzosa Espina, P. (1996), Aproximación a la medición del bienestar social, Secretariado de Publicaciones e Intercambio Científico, Universidad de Valladolid, España.

Zimbalist, A. (1989), “Crecimiento con equidad: el desarrollo cubano en una perspectiva comparada", Cuadernos de Nuestra América, núm. 13, s.p, julio-diciembre, La Habana.

Enviado: 7 de octubre de 2003. Aceptado: 4 de diciembre de 2005.

Francisco Ángel Becerra Lois tiene el doctorado en ciencias económicas. Es decano en la Facultad de Ciencias Económicas y Empresariales de la Universidad de Cienfuegos "Carlos Rafael Rodríguez". Su línea actual de investigación se centra en el desarrollo regional y local en la provincia de Cienfuegos, Cuba. Entre sus últimas publicaciones pueden contarse: 1) "Características subyacentes en el desarrollo socioeconómico de la provincia Cien- 
fuegos durante el periodo 1987-2000, Economía y Desarrollo, vol. 2, 2003; 2) "Desarrollo regional y cultura empresarial en un contexto de globalización”, Anuario de la Universidad de Cienfuegos, 2002, y 3) "El espacio regional como escenario de los procesos de globalización. La región ante el nuevo milenio", Memorias del IV Encuentro de Globalización y Problemas del Desarrollo, Palacio de Convenciones, La Habana, 2002.

Jesús René Pino Alonso es doctor en ciencias económicas. Se desempeña como jefe del Departamento de Estudios Económicos de la Facultad de Ciencias Económicas y Empresariales de la Universidad de Cienfuegos "Carlos Rafael Rodríguez". El desarrollo regional y local en la provincia Cienfuegos, Cuba, es su línea actual de investigación. Últimas publicaciones: 1) Cooperativismo, tres análisis jurídicos, Universidad de Cienfuegos Carlos Rafael Rodríguez, 1999, con Avelino Fernández Peiso y otros; 2) "Pobreza y desarrollo social", Fundación Universitaria Luis Amigó, vol. 1, año 1, núm. 2, con Clara Inés Orrego Correa y Fidel Márquez Sánchez, y 3) "La Universidad y sus perspectivas”, Estudios, Universidad de Especialidades del Espíritu Santo, núm. 2, Guayaquil, Ecuador, 1999, con Clara Inés Orrego Correa y Fidel Márquez Sánchez. 